\title{
Ocular symptoms as the initial clinical manifestations in patients with extraocular tumors
}

\author{
Jianlong Zheng ${ }^{1}$, Weiqi Chen ${ }^{1}$, Dingguo Huang ${ }^{1}$, Yifan Wang ${ }^{1}$, Dezhi Zheng ${ }^{1}$, Lingling Zhou ${ }^{1}$, \\ Marten E. Brelén ${ }^{2}$, Zijing Huang ${ }^{\wedge} \wedge$
}

${ }^{1}$ Joint Shantou International Eye Center of Shantou University and The Chinese University of Hong Kong, Shantou, China; ${ }^{2}$ Department of Ophthalmology and Visual Sciences, Chinese University of Hong Kong, Hong Kong, China

Contributions: (I) Conception and design: W Chen; (II) Administrative support: W Chen; (III) Provision of study materials or patients: J Zheng, Z Huang; (IV) Collection and assembly of data: J Zheng, Z Huang; (V) Data analysis and interpretation: Z Huang, W Chen, D Huang, Y Wang, D Zheng; (VI) Manuscript writing: All authors; (VII) Final approval of manuscript: All authors.

Correspondence to: Zijing Huang. Joint Shantou International Eye Center of Shantou University and The Chinese University of Hong Kong, 69 North Dongxia Rd, Shantou 515041, China. Email: huangzj@jsiec.org.

Background: Due to the variety of clinical presentation, some tumors may be concealed and easily misdiagnosed, leading to delays in management. We report a series of patients who initially presented to an Ophthalmic Clinic with ocular symptoms and were subsequently diagnosed with extraocular tumors.

Methods: Patients who presented to the ophthalmic outpatient clinic at the Joint Shantou International Eye Center with ocular symptoms between April 2013 and December 2019 and were subsequently diagnosed with intracranial or systemic tumors were reviewed retrospectively. Clinical data, including ocular symptoms and signs, ophthalmic and systemic imaging examinations, and the results of tumor biopsies were collected and analyzed.

Results: Twenty-three patients were included in this study, of which 16 were female $(69.6 \%)$ and 7 were male $(30.4 \%)$. Chief complaints at the first visit included visual loss $(n=20)$, proptosis $(n=2)$, and diplopia $(\mathrm{n}=1)$. Ocular examination revealed disc pallor $(\mathrm{n}=8)$ and swelling $(\mathrm{n}=3)$, choroidal mass with or without chorioretinal detachment $(\mathrm{n}=5)$, and proptosis $(\mathrm{n}=2)$. Visual field (VF) examination was performed in 11 patients of which hemianopia $(n=4)$ and non-specific field loss $(n=7)$ were noted. Brain CT or MRI, together with histopathological findings from surgical biopsies confirmed the diagnosis of the intracranial tumors in 18 cases, including pituitary adenoma $(n=7)$, meningioma $(n=2)$, oligodendroglioma $(n=1)$, sellar tumor $(n=1)$, suprachiasmatic arteriovenous aneurysm $(n=1)$, orbital glioma $(n=1)$, multiple intracranial tumors $(n=1)$, and sphenoid ossifying fibroma $(n=1)$. Nasopharyngeal carcinoma (NPC) $(n=3)$ was diagnosed with brain MRI and nasal endoscopy. Five patients were confirmed as choroidal metastasis secondary to lung cancer $(n=3)$, hepatoma ( $\mathrm{n}=1)$, and breast cancer $(\mathrm{n}=1)$.

Conclusions: Patients with extraocular tumors may present initially to an ophthalmologist with ocular symptoms. It is important to identify and appropriately manage these patients to avoid unnecessary delays in future treatment.

Keywords: Extraocular tumors; eye clinic; first visit; neuro-ophthalmology

Submitted Jan 19, 2021. Accepted for publication Mar 11, 2021.

doi: $10.21037 /$ atm-21-830

View this article at: http://dx.doi.org/10.21037/atm-21-830

^ ORCID: Weiqi Chen, 0000-0003-2852-8192; Zijing Huang, 0000-0003-2909-2538. 


\section{Introduction}

Extraocular tumors may cause severe ocular symptoms depending on the site of the primary lesion (1-3). Extraocular tumors refer to primary tumors located outside the eyeball, mainly including intracranial tumors, as well as tumors involving the nasopharynx, lung, breast, liver, and other organs in the body (2). Due to space occupying compression of the visual pathway, intracranial tumors typically cause optic nerve dysfunction, presenting with the signs of papilledema or optic atrophy and symptoms such as visual loss and visual field (VF) defects (4). Other neurological complications such as severe headache, projectile vomiting, and disturbance of consciousness, may arise if the extraocular tumor also causes a rise in intracranial pressure (5). In addition, dysphagia and dysarthria may occur if the tumor causes cranial nerve palsies (6). Some orbital tumors, such as an infiltrating nasopharyngeal carcinoma (NPC), may compress the eyeball, leading to proptosis and ocular motility disorders as the presenting feature (1-3). The choroid of the eye can often be a site of metastatic spread from extraocular tumors via a hematogenous route because of its abundant vasculature. Most commonly, a choroidal metastasis would spread from a primary tumor in the lung or breast (7) and since the patient may be asymptomatic from the primary tumor, the choroidal metastasis may be the primary presentation. Due to the variety of clinical presentations, an extraocular tumor may be misdiagnosed leading to delays in management and the early detection and diagnosis of these extraocular tumors is important to ensure prompt treatment is initiated to avoid further complications. In this study, we report a series of cases where an extraocular tumor initially presented with ophthalmic manifestations and highlight the important features that should alert an ophthalmologist to urgently investigate and refer the patient. We present the following article in accordance with the STROBE reporting checklist (available at http://dx.doi.org/10.21037/atm-21830).

\section{Methods}

\section{Subjects}

This retrospective study conformed to the guidelines of the Declaration of Helsinki (as revised in 2013) and was approved by the review board of the Ethics Committee of the Joint Shantou International Eye Center (JSIEC). Informed consent was exempt due to the retrospective nature of the study. The medical records of patients presenting between April 2013 and December 2019 and who met the following criteria were included: (I) Initially referred to an eye clinic in the Joint Shantou International Eye Center due to ocular symptoms without a known history of systemic tumors at presentation; (II) subsequently diagnosed as having intracranial or other systemic tumors confirmed by imaging and biopsy results in general hospitals. The exclusion criteria included any of the following: (I) diagnosed with primary ocular tumors or having other ocular diseases not relating to an extraocular tumor; (II) a history of intracranial and systemic tumors before presenting to the eye clinic.

\section{Ophthalmic examination}

Details of the clinical history and examination findings taken at the first visit were recorded. Ocular examinations including best-corrected visual acuity (BCVA) measurement with standard Snellen visual acuity chart, intraocular pressure (IOP) by non-contact tonometry, and dilated fundus examinations, were performed on all patients. Other ophthalmic findings including those of a VF test for patients with visual loss and disc pallor $(\mathrm{n}=11)$, fluorescence fundus angiography (FFA) for those with choroidal mass $(\mathrm{n}=5)$, and visual evoked potential (VEP) for patients with disc swelling $(n=4)$ were also recorded, although four patients were unable to perform the VF test due to poor visual function bilaterally. BCVA was converted to the log of the minimum angle of resolution for statistical purposes. As previously published, count finger was assigned as $1.85 \log$ MAR, hand motion as $2.30 \log M A R$, and light perception as 2.60logMAR (8).

\section{Systemic examinations and surgical interventions}

All patients included in this study were examined by a general physician at the general hospital. Radiological imaging, including brain magnetic resonance imaging (MRI) and/or computed tomography (CT), and orbital MRI and/or CT, were performed in patients with suspected intracranial tumors, and chest X-ray and CT, and abdominal color Duplex ultrasonography were performed in those with suspected systemic tumors. Nasal endoscopy was conducted in three patients in addition to the radiological imaging for further confirmation of an NPC. Information on surgical interventions and histopathological results from biopsies were also recorded. Ten patients with intracranial tumors 
Table 1 Clinical data in patients with extraocular tumors

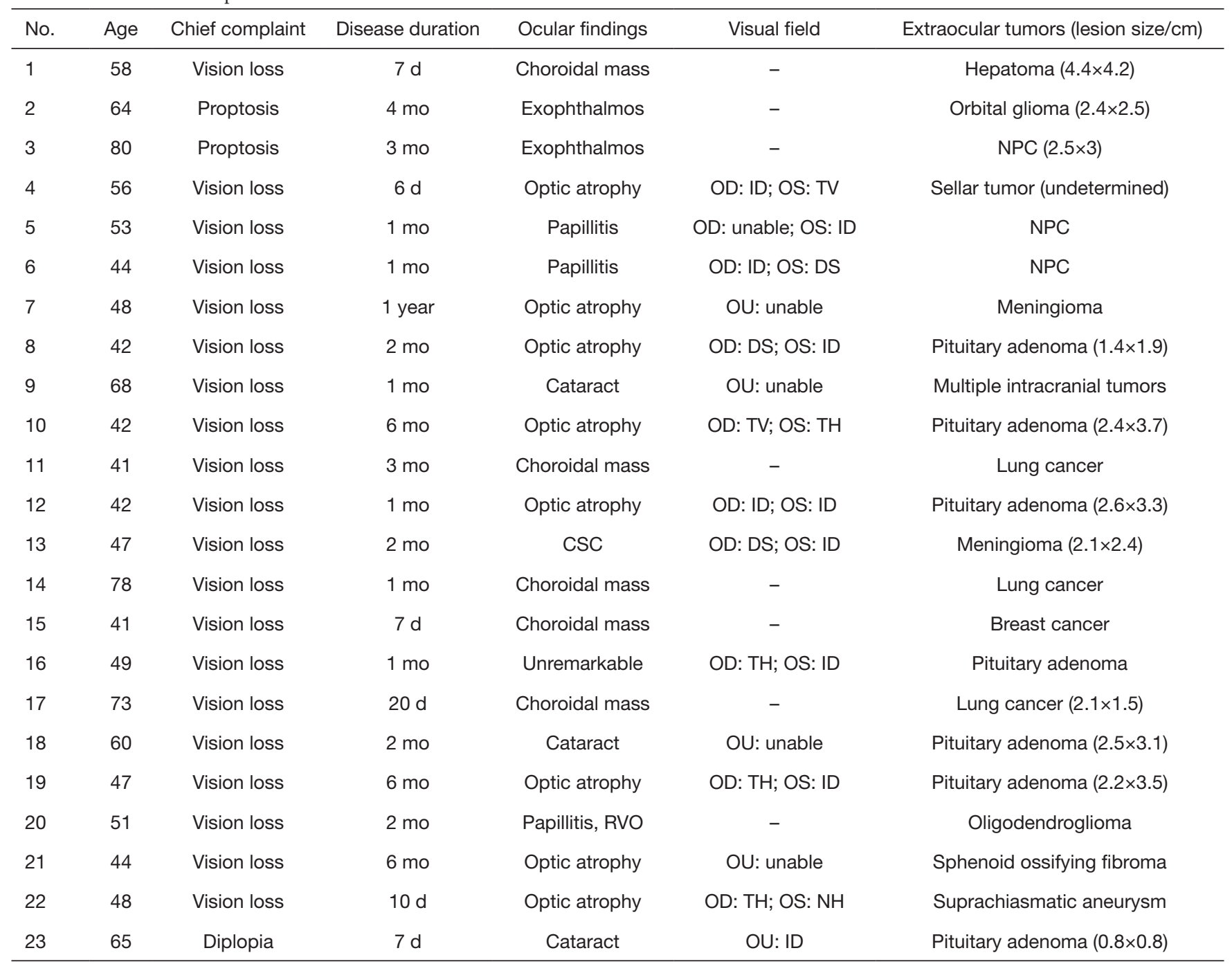

BCVA, best-corrected visual acuity converted to log of the minimum angle of resolution; RD, retinal detachment; CSC, central serous chorioretinopathy; RVO, retinal vein occlusion; NPC, nasopharyngeal carcinoma; ID, irregular defect; DS, decreased sensitivity; TV, tunnel vision; $\mathrm{TH}$, temporal hemianopsia; $\mathrm{NH}$, nasal hemianopsia; OU, both eyes; OS, left eye; OD, right eye; mo, months.

received surgical operations to remove the tumors and their types, sizes, and locations were determined. Radiotherapy was conducted in those patients with NPC and conservative treatment was given to all other patients. The definite diagnosis of the tumor was made according to radiological findings and pathological results from surgical biopsies.

\section{Statistical analysis}

Statistical analysis was performed using SPSS 18.0 software (SPSS Inc., Chicago, USA). Quantitative data were present as mean \pm standard deviation (SD). BCVA was converted to $\log$ MAR before statistical analysis.

\section{Results}

\section{Demographics}

A total of 23 patients were included, of which seven were male $(30.4 \%)$ and 16 were female $(69.6 \%)$ and the mean age at presentation was $54.0 \pm 12.2$ years. Baseline data are list in Table 1. 

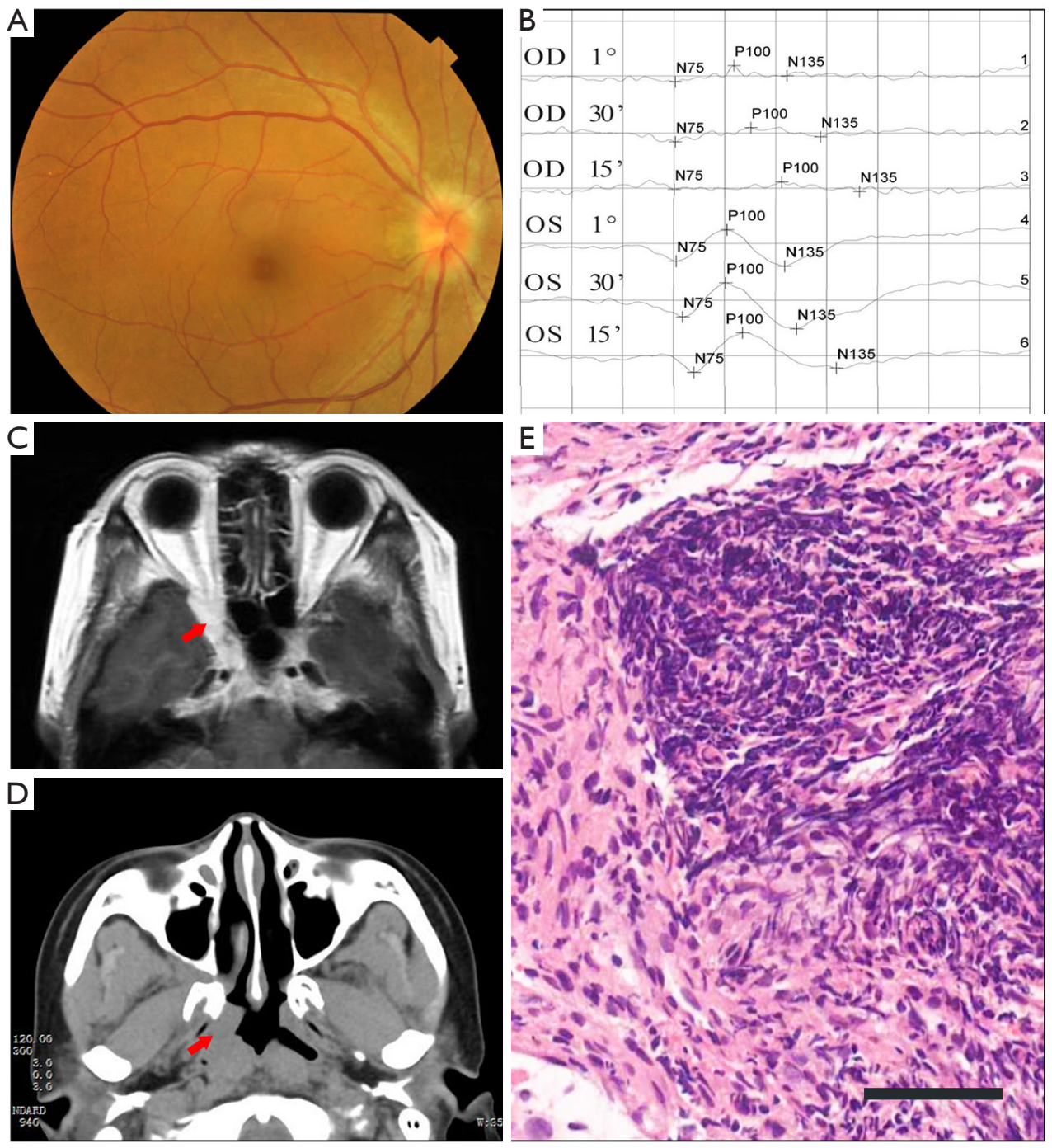

Figure 1 Clinical findings in a patient with nasopharyngeal carcinoma. (A) Ocular fundus photography showed acute papillary hyperemia and edema; (B) VEP test revealed prolonged latency and reduced amplitude in the right eye; (C) MRI imaging showed a mass located in the orbital apex and top of the nasopharynx with intermediate T1 signal that could be enhanced (arrow indicated); (D) the mass in the same place was observed by CT scanning (arrow indicated); (E) histopathological examination confirmed the diagnosis of nasopharyngeal carcinoma. hematoxylin-eosin staining, 200×. Scale bar: $50 \mu \mathrm{m}$. VEP, visual evoked potential.

\section{Ocular findings in patients with extraocular tumors}

Of note, vision loss was the most common presenting symptom at the first visit $(n=20,87.0 \%)$, while eyelid swelling and proptosis were the presenting features in two patients $(8.7 \%)$, and diplopia in one patient $(4.3 \%)$. The mean duration of presenting ocular symptoms in all individuals was $2.5 \pm 2.8$ months, with nearly half of the patients $(\mathrm{n}=12)$ complaining of acute vision loss for less than one month. At presentation, none of the patients complained of systemic symptoms, including but not limited to loss of weight, weakness, headache, vomiting, jaundice, hemoptysis, thoracodynia, coughing, dyspnea, or irregular menstruation. The BCVA in the affected eyes was $(1.14 \pm 0.90) \log$ MAR, ranging between $2.60 \log$ MAR (light perception) and $0.10 \log$ MAR $(20 / 25)$. IOP was normal in all individuals $(14.4 \pm 3.8 \mathrm{mmHg})$. On ocular examination, disc pallor was observed in eight eyes, whereas hyperemia and disc swelling were found in three (Figure 1). In addition, choroidal mass with or without chorioretinal 

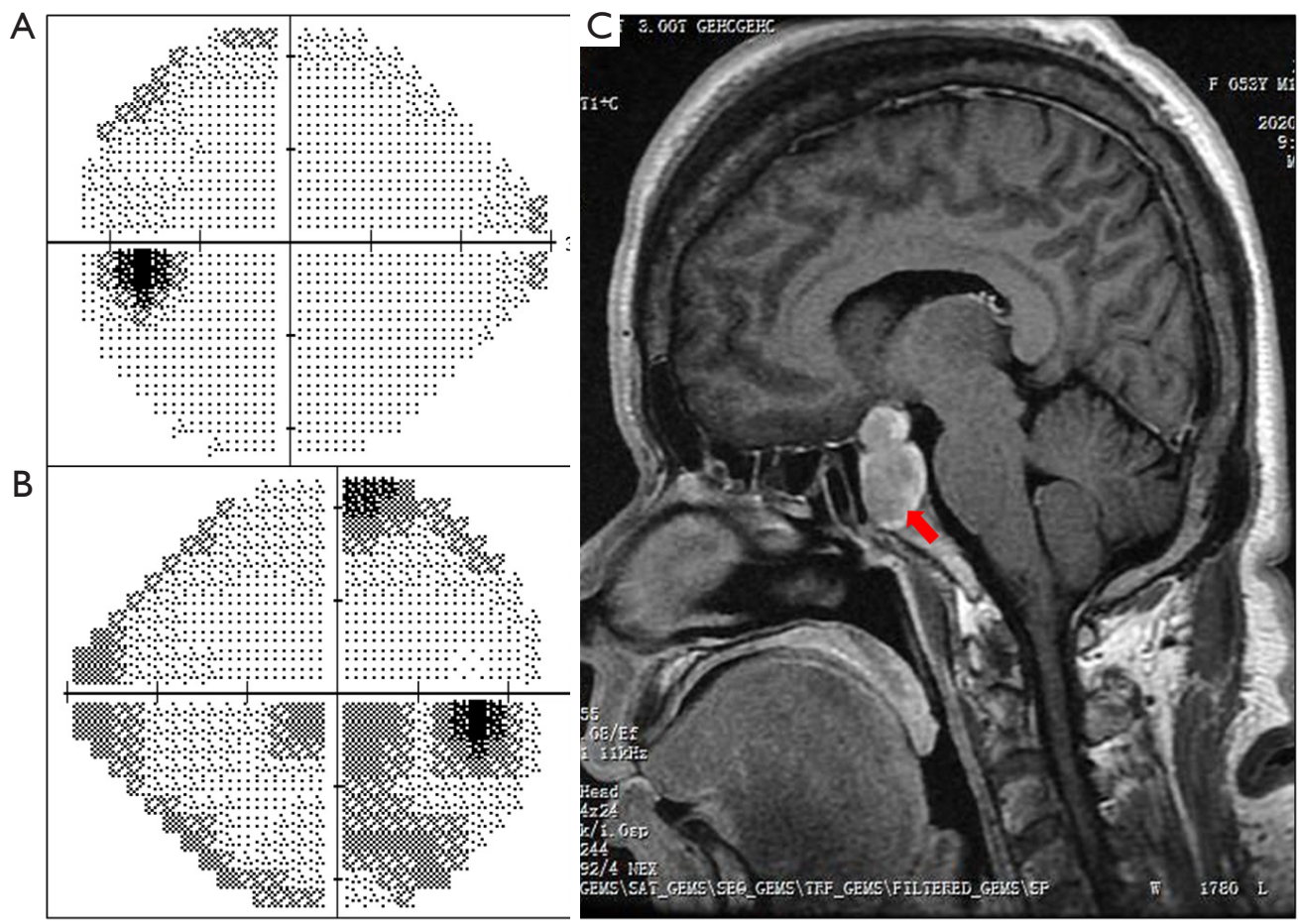

Figure 2 Clinical findings in a patient with pituitary adenoma. (A,B) Visual field test showed an irregular defect in the left eye (A) and incomplete hemianopsia in the right eye (B). (C) MRI scanning revealed a high-density mass presenting with the "hourglass sign" (arrow indicated).

detachment was seen in five eyes and proptosis was noted in two patients. Eleven patients underwent VF testing due to vision loss with disc pallor or swelling and among these, homonymous hemianopia was found in one patient and unilateral temporal hemianopia, combined with partial VF defect $(n=2)$ and tunnel vision $(n=1)$ in the contralateral eye, was found in three patients. Unilateral tunnel vision was detected in one patient and by contrast, early change or irregular defect in the VF pattern unilaterally or bilaterally was noted in six patients (Figure 2). Five patients with a choroidal mass with or without chorioretinal detachment received FFA examination and VEP was performed in four patients due to optic disc swelling. The results showed prolonged latency and reduced amplitude of the P100 response in the affected eyes.

\section{Diagnosis of extraocular tumors and follow-up information}

All patients were referred to the general hospital for systemic examination and a definitive diagnosis was made within a few weeks based on imaging examination and surgical biopsy results. Brain MRI and/or CT demonstrated intracranial tumors in 15 individuals, including pituitary adenoma $(\mathrm{n}=7)$, meningioma $(\mathrm{n}=2)$ (Figure 3), oligodendroglioma $(\mathrm{n}=1)$, sellar tumor $(\mathrm{n}=1)$, orbital glioma $(\mathrm{n}=1)$, suprachiasmatic arteriovenous aneurysm $(n=1)$, multiple intracranial tumors $(n=1)$, and sphenoid ossifying fibroma $(\mathrm{n}=1)$. NPC was confirmed in three patients through nasopharyngoscopy and MRI imaging. In addition, five patients with a choroidal mass received systemic examination (chest $\mathrm{CT}$ and $\mathrm{X}$-ray, abdominal CT and ultrasonography, and PET-CT) and were eventually diagnosed as primary lung cancer $(\mathrm{n}=3)$ (Figure 4), hepatoma $(\mathrm{n}=1)$, and breast cancer $(\mathrm{n}=1)$. The average tumor size as determined by CT or MRI imaging was $2.3 \mathrm{~cm} \times 2.7 \mathrm{~cm}$; the relatively small lesion size indicating the tumors were early stage. Surgical excision and biopsy were performed in 10 patients, and a final diagnosis with tumor classification then determined. Notably, four patients with pituitary tumors were followed up in the ophthalmic clinic several months after tumor excision, of which three showed improvement of their VF with maintained visual acuity. One patient had a reduction in visual function post-operatively. 

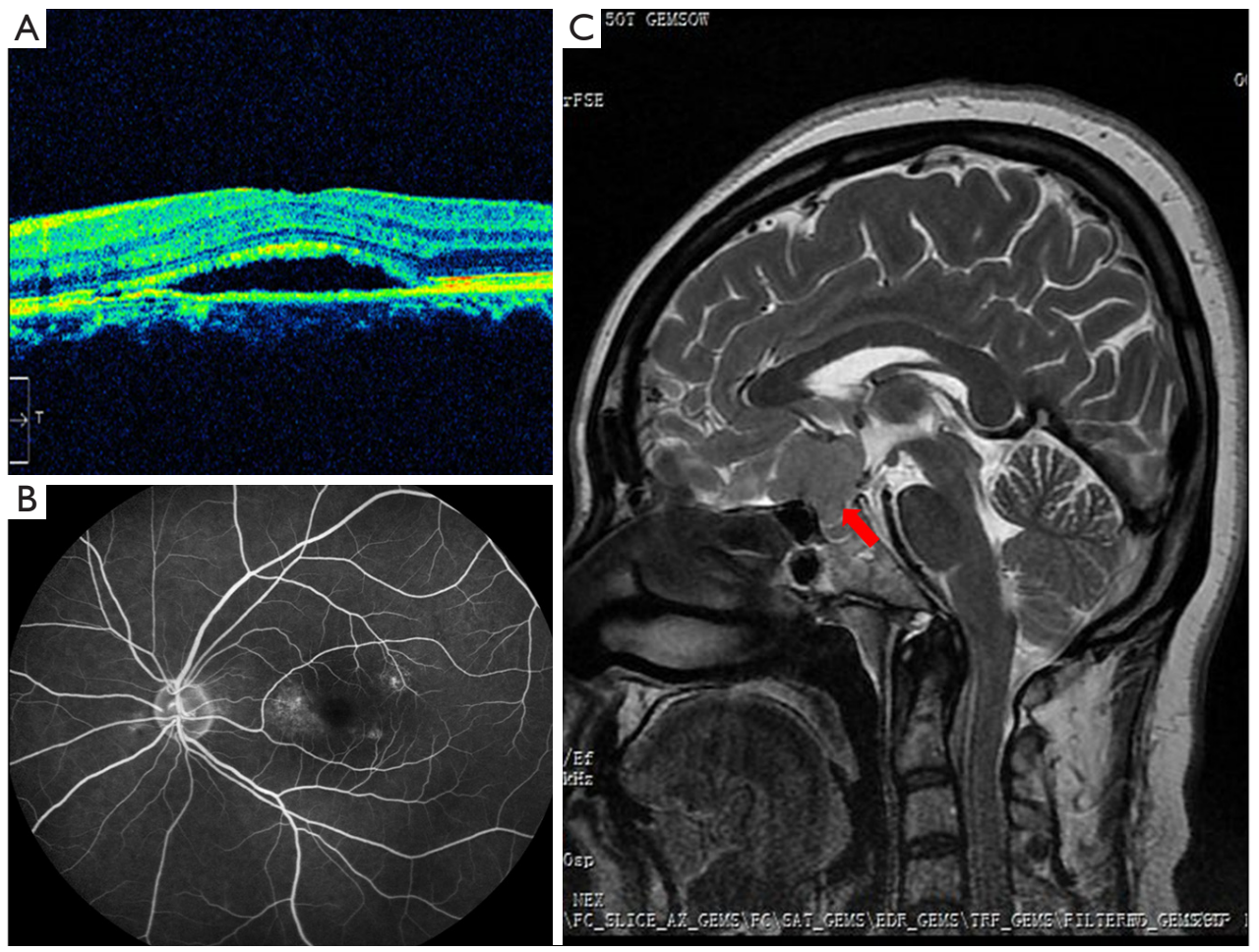

Figure 3 Images of a patient diagnosed with meningioma. (A,B) OCT and FFA exam revealed monocular serous neuroepithelial detachment of the central macular region. (C) MRI imaging showing an intermediate density soft tissue mass located in the suprasellar region (arrow indicated). OCT, optical coherence tomography; FFA, fluorescence fundus angiography.

\section{Discussion}

Patients with intracranial or systemic tumors may not initially experience neurological or systemic symptoms. Instead, their presenting complaint may be caused by the space occupying lesion compressing their visual pathway, leading to vision loss or other ocular symptoms $(9,10)$. Early detection and diagnosis of extraocular tumors are very important to start prompt treatment avoid further complications. In this study, we reviewed those patients with intracranial or other systemic tumors who initially presented with ocular symptoms to an ophthalmologist. The variety of presentations raises awareness about the importance of careful neuro-ophthalmic and systemic examination to avoid misdiagnosis and delayed treatment of extraocular tumors.

Intracranial tumors can cause progressive visual loss and VF defects long before the presence of neurological complications (3) and pituitary adenomas and meningiomas are the two most common types of adult intracranial tumors (10). Pituitary adenomas were the most prevalent tumor in this cohort of patients, being diagnosed in $30 \%$ of cases. This tumor typically presents with bitemporal hemianopia and will produce only mild endocrinological or intracranial hypertension symptoms in its early stages $(11,12)$, although paralysis of the cranial nerves and diplopia can sometimes develop due to parasellar extension of the mass $(13,14)$. Imaging examinations including brain CT and MRI are essential to determine the diagnosis. The most common clinical symptoms of meningiomas include headache, anosmia, and personality alteration (15), although in a few cases, larger meningiomas may infiltrate the frontal lobe and compress the optic chiasm leading to visual loss and VF defects (16). In this study, two patients were diagnosed with meningiomas and both patients were initially misdiagnosed due to their mild neurological symptoms and atypical VF defects.

At presentation, the hemianopia caused by an intracranial tumor can be asymmetrical, incomplete, or irregular (3). In this study, only one patient developed a typical symmetrical homonymous hemianopia due to a suprachiasmatic aneurysm, while a monocular hemianopia was seen in three patients and an incomplete hemianopia, irregular 

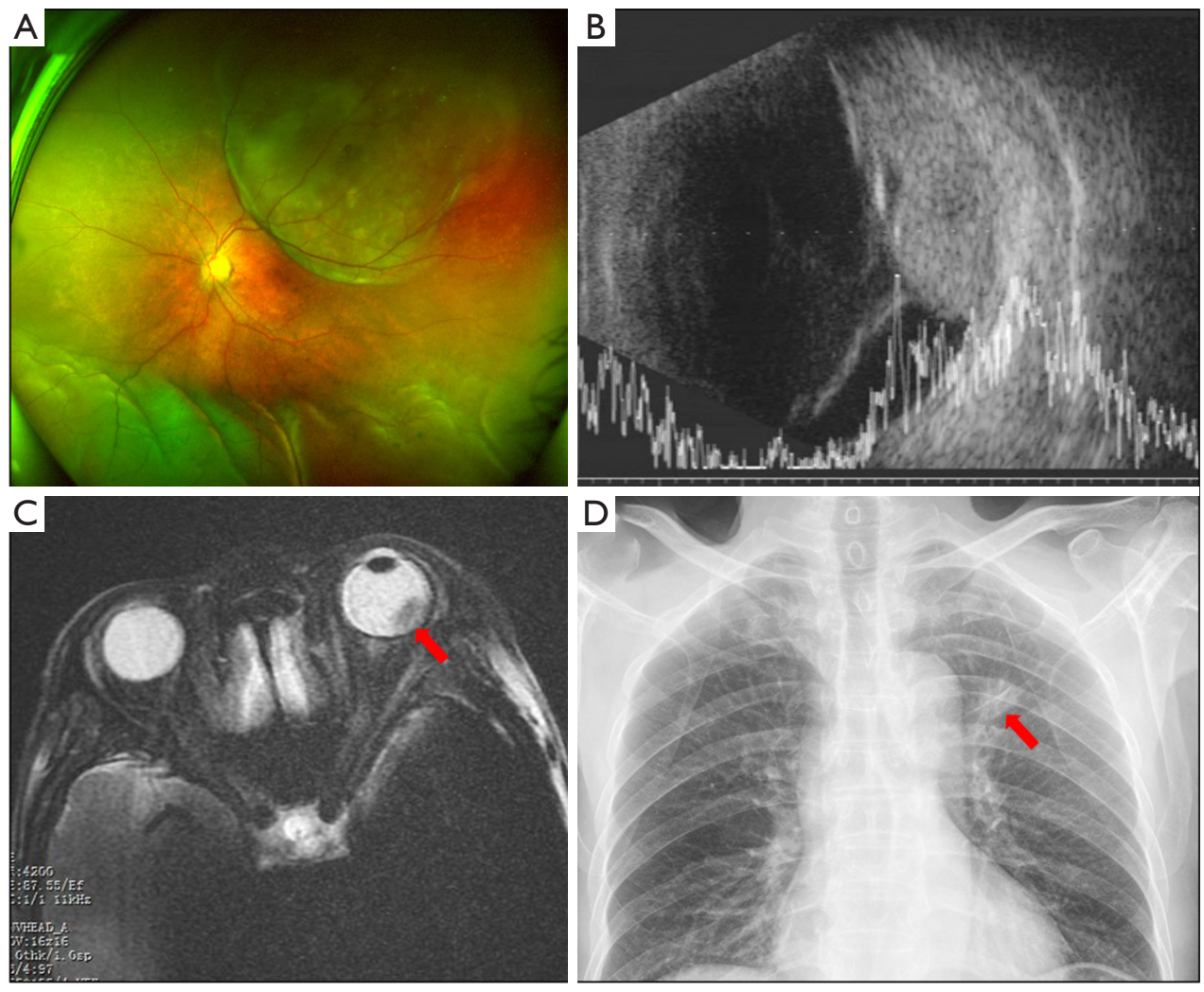

Figure 4 Images of a patient diagnosed with choroidal metastatic carcinoma secondary to lung cancer. (A,B) Scanning laser ophthalmoscopy (A) and B-scanning (B) showing a choroidal mass combined with inferior retinal detachment. (C) The mass (arrow indicated) exhibited a low and heterogeneous T2 signal in MRI imaging. (D) Chest X-ray revealed hyper-density masses (arrow indicated) in the left upper lung field.

VF defect, or tunnel vision was seen in six. Possible explanations for the atypical VF pattern may include: (I) the differences in location and size of the mass resulting in asymmetrical compression of the visual pathway; (II) early intracranial tumors which are small may result in only mild optic nerve compression, whereas large tumors may lead to tunnel vision due to long-term compression; and (III) in some patients the VF test was unreliable due to poor visual function. Patients with irregular or unusual VF loss should have an intracranial tumor considered as part of the differential diagnosis as frequently, tumor-induced VF defects and optic nerve atrophy are misdiagnosed as openangle glaucoma or high myopia, especially when the change of VF pattern is atypical (17). Careful assessment is needed for these patients at their first ophthalmic visit.

Tumor-associated ocular symptoms can also be attributed to compression from non-CNS tumors within the orbit. An NPC is an epithelial cancer originating from the nasopharynx, and is most prevalent in southern China and Southeast Asia (18). An enlarging NPC within the ethmoidal sinus can lead to proptosis and ocular motility disorders, and its compression can cause optic disc swelling and VF defects $(19,20)$. In clinical practice, NPC should be considered when patients present with vision loss and eye motility disorders, particularly when associated with nasal symptoms or cervical lymphadenopathy.

In this case series, five patients were diagnosed with a choroidal mass, with or without chorioretinal detachment, and B-scan and FFA demonstrated a space occupying lesions at the posterior wall of the eyeball. Chest X-ray and CT, abdominal ultrasonography, and hematological examinations confirmed the metastasis to be secondary to lung $(\mathrm{n}=3)$, breast $(\mathrm{n}=1)$, and liver $(\mathrm{n}=1)$. All patients presented with vision loss due to choroidal metastasis and none had symptoms associated with their primary tumor. As previously reported, intraocular metastases occur in over $10 \%$ of patients with systemic malignancies (21). The tumor can metastasize to the eye via the hematogenous 
route, and hence the posterior choroid is frequently the site of metastatic spread due to its abundant vasculature. A previous study of 84 patients with lung cancer showed that choroidal metastases occurred in $7.1 \%$ several months after the primary tumor was diagnosed (22). Another publication reported that in approximately one-third of patients, lung cancer was confirmed after the patient was diagnosed with a choroidal metastasis (23). In addition, choroidal metastases should not be mistaken for a choroidal melanoma, choroidal hemangioma, or central serous chorioretinopathy. Ocular examinations including FFA, B-scan and color doppler imaging, as well as systemic examinations such as chest $\mathrm{X}$-ray, CT, MRI, and tumor markers can be helpful in determining the diagnosis (24).

The sensitivity and specificity of CT and MRI in the diagnosis of intracranial tumors has previously been reported (25). The relatively small difference in density of some small tumors produces a larger intensity alteration on an MRI scan making this a preferable method compared to CT. In addition, MRI does not expose the patient to radiation, there is no requirement to use iodinated intravenous contrast agents, and there is good elimination of bone artifacts (25). One of our patients (case 6) suffered from an acute vision loss in her right eye. Ocular examinations showed disc swelling, irregular VF defects, and prolonged latency and reduced amplitude of the P100 VEP response, and an Orbital CT showed a spaceoccupying lesion at the inferior orbital fissure suggestive of a meningioma or glioma. However, enhanced MRI later demonstrated a lesion in the nasal pharynx which extended into the inferior orbital fissure through the foramen lacerum. The MRI had correctly identified the tumor to be an NPC which was later confirmed using electronic rhinolaryngoscopy. This demonstrates the superiority of MRI in detecting and identifying brain tumors.

It is also noted that six patients in this series were initially misdiagnosed by inexperienced young ophthalmologists, including choroidal metastases $(\mathrm{n}=2)$ misdiagnosed as central serous chorioretinopathy and exudative retinal detachment respectively, NPC $(n=1)$ misdiagnosed as acute optic neuritis, and intracranial tumors $(n=3)$ misdiagnosed as primary optic atrophy. The diagnosis was corrected at a subsequent visit by an experienced specialist. The possible reasons for misdiagnosis may include an incomplete inquiry to the history, incomprehensive consideration for the unexplainable vision loss, and a lack of specialized knowledge in neuro-ophthalmology.

\section{Conclusions}

We reported a series of patients who initially presented to an ophthalmic clinic with ocular symptoms and were finally diagnosed with an intracranial or systemic tumor. The manifestations of the tumors are varied and complicated due to their type, location, size, and growth. For cases with unknown vision loss, an intracranial tumor should be considered and further examinations including VF test and radiological imaging are necessary. Cases presenting with exudative retinal detachment or serous chorioretinopathy should be further investigated for choroidal metastasis with systemic imaging and tumor markers. This study highlights the importance for ophthalmologists to remain vigilant and thorough when evaluating patients to avoid any delay in the diagnosis and management of intracranial and systemic tumors.

\section{Acknowledgments}

Funding: None.

\section{Footnote}

Reporting Checklist: The authors have completed the STROBE reporting checklist. Available at http://dx.doi. org/10.21037/atm-21-830

Data Sharing Statement: Available at http://dx.doi. org/10.21037/atm-21-830

Conflicts of Interest: All authors have completed the ICMJE uniform disclosure form (available at http://dx.doi. org/10.21037/atm-21-830). The authors have no conflicts of interest to declare.

Ethical Statement: The authors are accountable for all aspects of the work in ensuring that questions related to the accuracy or integrity of any part of the work are appropriately investigated and resolved. This retrospective study conformed to the guidelines of the Declaration of Helsinki (as revised in 2013) and was approved by the review board of the Ethics Committee of the Joint Shantou International Eye Center (JSIEC). Informed consent was exempt due to the retrospective nature of the study.

Open Access Statement: This is an Open Access article distributed in accordance with the Creative Commons 
Attribution-NonCommercial-NoDerivs 4.0 International License (CC BY-NC-ND 4.0), which permits the noncommercial replication and distribution of the article with the strict proviso that no changes or edits are made and the original work is properly cited (including links to both the formal publication through the relevant DOI and the license). See: https://creativecommons.org/licenses/by-nc-nd/4.0/.

\section{References}

1. Ogra S, Nichols AD, Stylli S, et al. Visual acuity and pattern of visual field loss at presentation in pituitary adenoma. J Clin Neurosci 2014;21:735-40.

2. Shields CL, Welch RJ, Malik K, et al. Uveal metastasis: clinical features and survival outcome of 2214 tumors in 1111 patients based on primary tumor origin. Middle East Afr J Ophthalmol 2018;25:81-90.

3. Sefi-Yurdakul N. Visual findings as primary manifestations in patients with intracranial tumors. Int J Ophthalmol 2015;8:800-3.

4. Huang WB, Zhang XL, Wang W, et al. Ocular manifestations of intracranial germinomas: three cases report and literature review. Chin Med J (Engl) 2012;125:2790-3.

5. Perkins A, Liu G. Primary Brain Tumors in Adults: Diagnosis and Treatment. Am Fam Physician 2016;93:211-7.

6. Cheour M, Mazlout H, Agrebi S, et al. Compressive optic neuropathy secondary to a pituitary macroadenoma. J Fr Ophtalmol 2013;36:e101-4.

7. Arepalli S, Kaliki S, Shields CL. Choroidal metastases: origin, features, and therapy. Indian J Ophthalmol 2015;63:122-7.

8. Schulze-Bonsel K, Feltgen N, Burau H, et al. Visual acuities "hand motion" and "counting fingers" can be quantified with the Freiburg visual acuity test. Invest Ophthalmol Vis Sci 2006;47:1236-40.

9. Shao S, Li X. Clinical features and analysis in $\mathbf{1 3 8 5}$ Chinese patients with pituitary adenomas. J Neurosurg Sci 2013;57:267-75.

10. Aui-aree N, Phruanchroen C, Oearsakul T, et al. Three years experience of suprasellar tumors in neuroophthalmology clinic. J Med Assoc Thai 2010;93:818-23.

11. Molitch ME. Diagnosis and treatment of pituitary adenomas: a review. JAMA 2017;317:516-24.

12. Levy A. Pituitary disease: presentation, diagnosis, and management. J Neurol Neurosurg Psychiatry 2004;75:iii47-iii52.
13. Saffra N, Kaplow E, Mikolaenko I, et al. Isolated sixth cranial nerve palsy as the presenting symptom of a rapidly expanding ACTH positive pituitary adenoma: a case report. BMC Ophthalmol 2011;11:4.

14. Lee JP, Park IW, Chung YS. The volume of tumor mass and visual field defect in patients with pituitary macroadenoma. Korean J Ophthalmol 2011;25:37-41.

15. Buerki RA, Horbinski CM, Kruser T, et al. An overview of meningiomas. Future Oncol 2018;14:2161-77.

16. Jung JJ, Warren FA, Kahanowicz R. Bilateral visual loss due to a giant olfactory meningioma. Clin Ophthalmol 2012;6:339-42.

17. Jonas JB, Nagaoka N, Fang YX, et al. Intraocular pressure and glaucomatous optic neuropathy in high myopia. Invest Ophthalmol Vis Sci 2017;58:5897-906.

18. Kamran SC, Riaz N, Lee N. Nasopharyngeal carcinoma. Surg Oncol Clin N Am 2015;24:547-61.

19. Xu Y, Lin G, Lin C, et al. Treatment and prognosis of nasopharyngeal angiofibroma involving the eye and optic nerve. J Laryngol Otol 2012;126:1108-13.

20. Ong Tone S, DeAngelis D, Monteiro E, et al. Nasopharyngeal carcinoma presenting with no light perception vision. Can J Ophthalmol 2016;51:e39-e40.

21. Sun T, Yin Y, Liu T, et al. SU-E-T-596: Choroidal metastases irradiation with volumetric modulated arc radiotherapy. Med Phys 2012;39:3843.

22. Kreusel KM, Wiegel T, Stange M, et al. Choroidal metastasis in disseminated lung cancer: frequency and risk factors. Am J Ophthalmol 2002;134:445-7.

23. Shields CL, Shields JA, Gross NE, et al. Survey of 520 eyes with uveal metastases. Ophthalmology 1997;104:1265-76.

24. Ye PP, Xu J, Su ZT, et al. The application of ultra-widefield fundus autofluorescence in early metastatic choroidal tumor screening. Int J Ophthalmol 2019;12:1978.

25. Newton TH, Kelly WM, Brant-Zawadzki M, et al. Comparison of Magnetic Resonance Imaging with Computed Tomography in the Detection and Characterization of Intracranial Tumors. Berlin Heidelberg: Springer, 1988:193-8.

(English Language Editor: B. Draper)

Cite this article as: Zheng J, Chen W, Huang D, Wang Y, Zheng D, Zhou L, Brelén ME, Huang Z. Ocular symptoms as the initial clinical manifestations in patients with extraocular tumors. Ann Transl Med 2021;9(6):497. doi: 10.21037/atm-21-830 